\title{
Glancing through the accretion column of EXO 2030+375
}

\author{
Carlo Ferrigno $^{1}$, Patryk Pjanka ${ }^{2,3}$, Enrico Bozzo ${ }^{1}$, Dmitry Klochkov ${ }^{4}$, Lorenzo Ducci ${ }^{1,4}$, and Andrzej A. Zdziarski ${ }^{3}$ \\ 1 ISDC, Department of astronomy, University of Geneva, chemin d'Écogia 16, 1290 Versoix, Switzerland \\ e-mail: carlo.ferrigno@unige.ch \\ 2 Department of Astrophysical Sciences, Princeton University, 4 Ivy Lane, NJ 08544 Princeton, USA \\ 3 Nicolaus Copernicus Astronomical Center, Polish Academy of Sciences, Bartycka 18, 00-716 Warszawa, Poland \\ 4 Institut für Astronomie und Astrophysik, Kepler Center for Astro and Particle Physics, Eberhard Karls Universität, Sand 1, \\ 72076 Tübingen, Germany
}

Received 25 November 2015 / Accepted 12 July 2016

\begin{abstract}
Context. The current generation of X-ray instruments progressively reveals more and more details about the complex magnetic field topology and the geometry of the accretion flows in highly magnetized accretion-powered pulsars.

Aims. We took advantage of the large collecting area and good timing capabilities of the EPIC cameras onboard XMM-Newton to investigate the accretion geometry onto the magnetized neutron star hosted in the high-mass X-ray binary EXO 2030+375 during the rise of a source type I outburst in 2014.

Methods. We carried out a timing and spectral analysis of the XMM-Newton observation as a function of the neutron star spin phase. We used a phenomenological spectral continuum model comprising the required fluorescence emission lines. Two neutral absorption components are present: one covering the source fully, one only partially. The same analysis was also carried out on two Suzaku observations of the source performed during outbursts in 2007 and 2012, to search for possible spectral variations at different luminosities.

Results. The XMM-Newton data caught the source at an X-ray luminosity of $2 \times 10^{36} \mathrm{erg} \mathrm{s}^{-1}$ and revealed a narrow dip-like feature in its pulse profile that was never reported before. The width of this feature corresponds to about one hundredth of the neutron star spin period. Based on the results of the phase-resolved spectral analysis we suggest that this feature can be ascribed to the self-obscuration of the accretion stream passing in front of the observer line of sight. We inferred from the Suzaku observation carried out in 2007 that the self-obscuration of the accretion stream might produce a significantly wider feature in the neutron star pulsed profile at higher luminosities $\left(\gtrsim 2 \times 10^{37} \mathrm{erg} \mathrm{s}^{-1}\right)$.

Conclusions. This discovery allowed us to derive additional constraints on the physical properties of the accretion flow in this object at relatively small distances from the neutron star surface. The narrow dip-like feature in the pulse profile is so far unique among all known high mass X-ray binaries hosting strongly magnetized neutron stars.
\end{abstract}

Key words. X-rays: binaries - stars: neutron - pulsars: individual: EXO 2030+375

\section{Introduction}

The source EXO 2030+375 is a prototypical high-mass Be X-ray binary (BeXRB), comprising a neutron star (NS) and a Be companion. X-ray outbursts are generally produced when the orbit of the pulsar intercepts the companion's decretion disk. Be/X-ray binaries typically show two types of outbursts: (1) giant outbursts (type II), which last tens of days and are characterized by high luminosities and high spin-up rates (i.e., a significant increase in pulse frequency); and (2) normal outbursts (type I), which are characterized by lower luminosities and less pronounced spin-up rates (if any). Type I outbursts are known to occur (almost) regularly at each periastron passage in some systems (Stella et al. 1986; Bildsten et al. 1997). During these events, the material lost by the Be star is first focused toward the NS as a consequence of its strong gravitational field and is then funneled by its intense magnetic field $\left(B \sim 10^{12-13} \mathrm{G}\right)$ down to the magnetic poles, where one or more accretion columns are formed.

The bulk of the continuum X-ray emission from BeXRBs is produced within the accretion columns by the Compton scattering of seed thermal photons from the hot spot on the NS surface or by bremsstrahlung processes occurring along the column
(Becker \& Wolff 2007, and references therein). The spectral energy distribution of these sources is expected to show a remarkable dependence on the spin phase that is due to the changes in the viewing angle of the observer and the angular dependence of the Compton scattering cross section in a strong magnetic field (Meszaros \& Nagel 1985; Meszaros \& Riffert 1988). Iron fluorescence lines corresponding to different ionization levels of these heavy ions are commonly observed in BeXRBs and ascribed to the illumination of the accreting material at different distances from the NS by the intense X-ray radiation.

The broadband spectra of BeXRBs are commonly described by different phenomenological models, the most widely used one being an absorbed power-law modified at high energy by an exponential cutoff. Depending on the statistics of the data and the energy coverage, it proved necessary in several cases to complement these relatively simple spectral models with the additions of broad Gaussian components (see, e.g., Klochkov et al. 2007; Suchy et al. 2008) and/or partial covering absorbers (Naik et al. 2011, 2013; Naik \& Jaisawal 2015). Furthermore, the cyclotron resonant scattering of electrons in the high magnetic field of the NS is known to produce characteristic absorption lines that have been observed in several of these systems and included in the 
spectral fits by using either Gaussian or Lorentzian profiles (see, e.g., Walter et al. 2015, for a recent review). Cyclotron resonant scattering features (CRSFs) can be used to infer the NS surface magnetic field strength as the centroid energy of the fundamental line is at $E_{\text {cyc }} \simeq 11.6 B_{12} \times(1+z)^{-1} \mathrm{keV}$, where $B_{12}$ is the NS magnetic field strength in units of $10^{12} \mathrm{G}$ and $z$ the redshift of the scattering medium.

The source EXO 2030+375 hosts a $42 \mathrm{~s}$ pulsar discovered with EXOSAT during a giant type II outburst in 1985 (Parmar et al. 1989). The compact object orbits a B0 Ve star (Coe et al. 1988, and references therein) every 46 days (Wilson et al. 2005, 2008). The estimated distance to the source is $7.1 \mathrm{kpc}$ (Wilson et al. 2002). Type I outbursts reaching peak fluxes of about $100 \mathrm{mCrab}(15-50 \mathrm{keV})$ have regularly been detected from EXO $2030+375$ at virtually all periastron passages since 1991 (the outburst peak typically occurs about $\sim 7 \mathrm{~d}$ after the periastron passage; see, e.g., Wilson et al. 2005). In the period spanning from 1992 to 1994, the type I outbursts have been brighter than average and the NS showed a remarkable spin-up. From 1994 to 2002, the outbursts showed somewhat lower peak luminosities (a factor of few), and the pulsar displayed a clear spin-down trend. A new re-brightening period followed until June 2006, when EXO 2030+375 underwent its second observed giant type II outburst. After a number of binary orbits characterized by a higher persistent luminosity than average and type I outburst achieving a peak flux of 200-300 mCrab (15-50 keV), the source returned to its normal behavior.

During type I and type II outbursts, the broadband spectrum of EXO $2030+375$ can be reasonably well described by using an absorbed $\left(N_{\mathrm{H}} \simeq 10^{22} \mathrm{~cm}^{-2}\right)$ power law with a high-energy exponential roll-over. Contrasting results have been published concerning the presence of possible CRSFs in the X-ray emission from the source. Reig \& Coe (1999) reported on the possible detection of such a feature with a centroid energy of $\sim 36 \mathrm{keV}$, while Wilson et al. (2008) found evidence of a CRSF at $\sim 11 \mathrm{keV}$. Klochkov et al. (2007) showed, however, that the data used by Wilson et al. (2008) could also be reasonably well characterized without the cyclotron line by including a broad Gaussian emission feature at $\sim 15 \mathrm{keV}$ in the fit (as observed in other high-mass X-ray binaries; see, e.g., the discussion in Ferrigno et al. 2009, and references therein). Klochkov et al. (2008) also reported on the detection of a CRSF at $\sim 63 \mathrm{keV}$ in spin-resolved spectra extracted during the peak of the 2006 giant outburst. This could correspond to a higher harmonics of the previously suggested cyclotron line at $\sim 36 \mathrm{keV}$.

The pulse profile of EXO 2030+375 is known to be strongly dependent on the X-ray luminosity. At the peak of the outbursts its characteristic shape is generally interpreted in terms of a fan beam-like emission, while it becomes more reminiscent of what is expected in the case of a pencil beam emission during the decay of the outburst (Parmar et al. 1989; Klochkov et al. 2008). A similar interpretation was also suggested in the detailed study carried out by Sasaki et al. (2010) with a pulse decomposition method.

In this paper, we report on the first XMM-Newton observation of EXO $2030+375$ performed during the rise of a type I outburst in 2014. The large collecting area and good timing resolution of the EPIC-pn camera onboard XMM-Newton allowed us to extract the source pulse profiles with more than 100 phase bins, revealing a peculiar sharp and deep feature never detected before (Sect. 3). Following our spectral results (Sect. 4), we suggest that this feature is caused by the obscuration effect of the accretion stream passing in front of the observer line of sight to the source (Sect. 5). The implications of our results are discussed in Sect. 6 and summarized in Sect. 7.

\section{Observations and data analysis}

We first analyze the XMM-Newton observation of EXO 2030+375, which caught the source during the rise to the peak of a type I outburst in 2014. The results of this observation are then compared with those obtained with Suzaku during type I X-ray outbursts that occurred in 2007 and 2012. A $\log$ of all observations is provided in Table 1.

\subsection{XMM-Newton}

During the XMM-Newton observation of EXO 2030+375 in 2014 the EPIC-pn was operated in timing mode, while the MOS1 was in small window and the MOS2 in full frame. We followed standard data pipeline reduction procedures (epchain, emchain, rgsproc) by using the SAS v.13. No episodes of enhanced solar activity were revealed in the data, and thus we retained the full exposure available for all EPIC cameras. We extracted the EPIC-pn spectra and light curves of the source (background) from the CCD columns 34-43 (3-10). The average source count rate measured by the EPIC-pn was of $31 \mathrm{cts} / \mathrm{s}$, thus pile-up was not an issue for these data. The MOS data suffered instead a significant pile-up. To correct for this issue, we removed a progressively larger central part of the MOS point spread function for the source spectral extraction until a good match was obtained with the measured flux and spectral shape of the EPIC-pn data. Data from the MOS1 could be relatively well corrected by extracting the source spectra from an annular region with an inner radius of 150 pixels $(1.125 \mathrm{arcmin})$ and an external radius of 1750 pixels (1.46 arcmin). The MOS 1 background spectrum was extracted by using an equivalently large region from a different chip not contaminated by the source X-ray emission. The pile-up of the MOS2 was too severe to attempt any correction, and we therefore discarded these data from further analysis. All EPIC spectra of the source were optimally rebinned using the prescription in paragraph 5.4 of Kaastra \& Bleeker (2016). The RGS spectra of the first dispersion order were characterized by a low signal-to-noise ratio $(\mathrm{S} / \mathrm{N})$ and were therefore combined with the tool rgscombine. The spectra were then rebinned with a minimum number of 20 photons per energy bin. We did not make use of the second-order spectra because of their significantly lower $\mathrm{S} / \mathrm{N}$.

A first look at the source EPIC and RGS spectra revealed that they are heavily absorbed below $1 \mathrm{keV}$. As reported in a number of other published papers in the literature, we noted that the EPIC-pn data in timing mode suffered redistribution issues below $1.7 \mathrm{keV}$ with this high absorption column density. Significant discrepancies between the residuals in the EPIC-pn, MOS1, and the RGS appeared below this energy for any fit we attempted. For the final scientific analysis, we therefore limited our fits to the energy range $1.7-11 \mathrm{keV}$ for the EPIC-pn, $1-10 \mathrm{keV}$ for the MOS1, and 1-2.1 keV for the two RGSs. We also excluded the EPIC-pn data in the energy range $2.19-2.39 \mathrm{keV}$ from the fits because of the instrumental residuals related to the edge that is produced by gold in the effective area. This issue is linked to small uncertainties in the energy calibration for the fast modes coupled with the large number of detected counts (see, e.g., Ferrigno et al. 2014, and references therein). 
Table 1. Log of all observations.

\begin{tabular}{lcccccc}
\hline \hline Orbital phase & Start time [UT] & Stop time [UT] & \multicolumn{2}{c}{ Exposures [ks] } & & $L_{\mathrm{X}}{ }^{a}[\mathrm{erg} / \mathrm{s}]$ \\
& & & EPIC-pn & EPIC-MOS 1 & RGS & \\
\hline $0.022-0.030$ & $2014-05-2923: 58$ & $2014-05-308: 30$ & 30.2 & 31.7 & 32.8 & $2.1 \times 10^{36}$ \\
\hline & & & XIS & HXD & \\
\hline $0.027-0.068^{b}$ & $2012-05-2320: 12$ & $2012-05-2518: 57$ & 77.9 & 67.7 & $2.7 \times 10^{36}$ \\
$0.132-0.159^{c}$ & $2007-05-1420: 37$ & $2007-05-1603: 45$ & 28.6 & 50.0 & $1.9 \times 10^{37}$ \\
\hline
\end{tabular}

Notes. ${ }^{(a)}$ In the $1-10 \mathrm{keV}$ band and assuming a distance of 7.1 kpc. ${ }^{(b)}$ Data set also analyzed in Naik \& Jaisawal (2015). ${ }^{(c)}$ Data set also analyzed in Naik et al. (2013).

\subsection{Suzaku}

We used the tools included in HEASoft v.6.14 and the latest calibration files available in CALDB for the HXD (13.09.2011) and the XIS (01.07.2014) for the entire Suzaku data analysis. The raw data from the two instruments were reprocessed using aepipeline separately for the XIS, the PIN, and the GSO detectors. The XIS data suffered a significant pile-up due to the brightness of the source, and we used pile_estimate. $s 1^{1}$ to evaluate the annular extraction region required to filter out all events affected by a pile-up fraction $\gtrsim 5 \%$. With this method, we reduced the data pile-up fraction from an original $\sim 8 \%$ to $\sim 2 \%$. The source and background spectra were derived from the cleaned and corrected event files using xselect and identical extraction annular regions. Spectra were grouped as described in the previous paragraph. We generated the redistribution matrix functions (RMFs) and auxiliary response functions (ARFs) with xisrmfgen and xissimarfgen, respectively. The non-X-ray background files (NXBs) were generated using xisnxbgen and combined with previously extracted backgrounds with mathpha (taking into account the proper scaling for the effective area). No NXB could be generated for the XIS1 detector because we lack data in the CALDB. Following the standard recommendations to avoid uncertainties in the effective area and response matrix of the XIS detectors, we considered only the energy range $1-10 \mathrm{keV}$ for the XIS0 and XIS3, and the energy range $1-8 \mathrm{keV}$ for the XIS1. The energy ranges $1.72-1.88 \mathrm{keV}, 2.19-2.37 \mathrm{keV}$, and $1.72-2.37 \mathrm{keV}$ were excluded for the XIS0, XIS3, and XIS1, respectively, because of the known $\mathrm{Au}$ and Si calibration features (Nowak et al. 2011; Kühnel et al. 2013). The background models and response files for the HXD, PIN, and GSO are supplied by the mission team ${ }^{2}$. We used the "tuned" version of these files for the PIN. The PIN and GSO spectra were extracted using hxdpinxbpi and hxdgsoxbpi.

\section{Timing analysis}

To carry out a proper timing analysis of the XMM-Newton data, we first converted the arrival time of each photon detected by the EPIC-pn and MOS1 to the solar system barycenter using the known optical position of the source (Cutri et al. 2003). The newly obtained arrival times were then converted into the system of the binary line of the nodes using the first solution for the orbital ephemeris of the source published in Table 2 of Wilson et al. (2002). The spin period of the source in the $X M M-N e w t o n$ data was then determined at $41.2858(8) \mathrm{s}$ (at $1 \sigma$

$\begin{array}{ll}1 & \text { http://space.mit.edu/ASC/software/suzaku/pest.html } \\ 2 & \text { http://wwW.astro.isas.jaxa.jp/suzaku/analysis/hxd/ }\end{array}$ c.l.) using an epoch-folding technique. This value is compatible with the Fermi/GBM measurement at the same epoch ${ }^{3}$.

Pulse profiles were extracted from the EPIC-pn and MOS1 data with 175 phase bins in three energy bands, chosen to equally split the number of photons. Their shape (see Fig. 1) is very similar to that observed by Suzaku in 2012 (see Naik et al. 2013, and our Fig. 8), when the source was at a comparable luminosity. Remarkable swings in the hardness ratio are present at phases $\lesssim 0.5$. Additionally, we noted in the EPIC-pn pulse profile a sharp Vlike feature at phase 0.27 (immediately before the main pulse profile peak) that was never reported before. A zoom of the data closer to the phase of this feature is presented in Fig. 2. Its profile displays a noticeable energy dependence, comprising an increased absorption in the soft X-rays at phases $\lesssim 0.27$ and an enhanced re-emission at harder X-rays. The peak of the reemission occurs at later phases for harder X-rays.

For the timing analysis of the 2007 and 2012 Suzaku data we followed the same procedures as in Naik et al. (2013) and Naik \& Jaisawal (2015), respectively. The derived pulse profiles are shown in Figs. 8 and 9 and fully agree with those published previously.

\section{Phase-averaged spectral analysis}

A number of sophisticated spectral models have been developed to describe the X-ray energy distribution of highly magnetized accreting X-ray pulsars (e.g., Becker \& Wolff 2007; Farinelli et al. 2012; Farinelli et al. 2016). They include a fairly detailed description of the physics of the NS accretion column, where the bulk of the X-ray emission is produced (see Sect. 1). In this paper, we focus on the analysis of the sharp feature highlighted in Sect. 3, as well as on any possible spectral change related to it, and thus we did not attempt to fit the XMM-Newton data of EXO 2030+375 with these models because the energy range of the EPIC cameras is too limited to provide significant constraints to all their free parameters. These models are not designed to perform spin phase-resolved spectral analysis, however. We therefore opted for a phenomenological description, comprising a power law modified by an exponential cutoff at high energy:

$N(E)= \begin{cases}E^{-\Gamma} & \text { for } E \leq E_{\mathrm{C}}, \\ E^{-\Gamma} \exp \left(-\frac{E-E_{\mathrm{C}}}{E_{\mathrm{F}}}\right) & \text { for } E>E_{\mathrm{C}} .\end{cases}$

In the equation above, $E_{\mathrm{C}}$ and $E_{\mathrm{F}}$ are the cutoff and folding energies, respectively. We used this model to fit for both the phaseaveraged and phase-resolved XMM-Newton and Suzaku spectra.

\footnotetext{
3 The Fermi/GBM measurements are available at the URL http: //gammaray.nsstc.nasa.gov/gbm/science/pulsars/ lightcurves/exo2030.html
} 


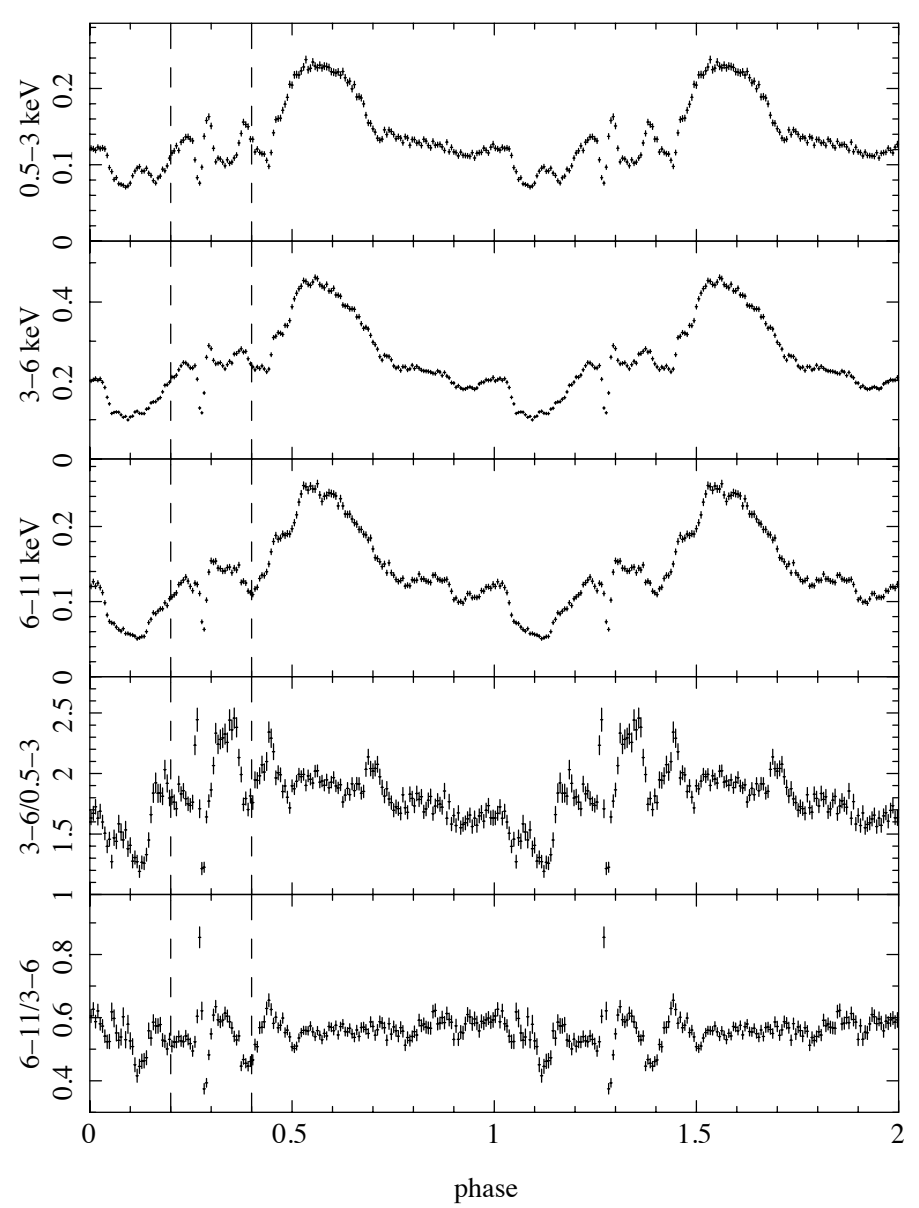

Fig. 1. Pulse profiles and hardness ratios extracted from the EPIC-pn data in the $0.5-3 \mathrm{keV}, 3-6 \mathrm{keV}$, and $6-11 \mathrm{keV}$ energy bands. The two lower panels shows the ratio between the counts in the hard and soft energy bands. Vertical dotted lines highlight the zoom region of Fig. 2.

We included in the spectral model a first absorption component (tbnew_feo) ${ }^{4}$, representing the summed contribution of the Galactic interstellar medium along the line of sight to the source and the local wind material from the massive companion surrounding the NS (100\% coverage). We also introduced a second, partially covering neutral absorption component (tbnew_pcf) to take into account the X-ray extinction that is due to material closer to the NS and/or within its accretion column. The parameters of this absorption component are the column density of the absorber $N_{\mathrm{H} \text {,partial }}$ and its covering fraction $f$ (which represents the fraction of the total radiation from the source that is affected by the presence of the absorbing material). We checked that the introduction of a redshift for the neutral absorber material, expected from the strong influence of the NS gravitational field on the radiation emerging close to the surface of the compact object, could not be reasonably constrained by our dataset. We note that this absorber is expected to be at least partly ionized because of the relatively high X-ray luminosity of the source. However, when we attempted to use a partially covering ionized absorber model (warmabs ${ }^{5}$ ), the fit revealed that the ionization parameter could only be constrained to be $\log _{10} \xi<-3$

\footnotetext{
4 http://pulsar.sternwarte.uni-erlangen.de/wilms/ research/tbabs/

5 http://heasarc.gsfc.nasa.gov/xstar/docs/html/ node $102 . \mathrm{html}$
}

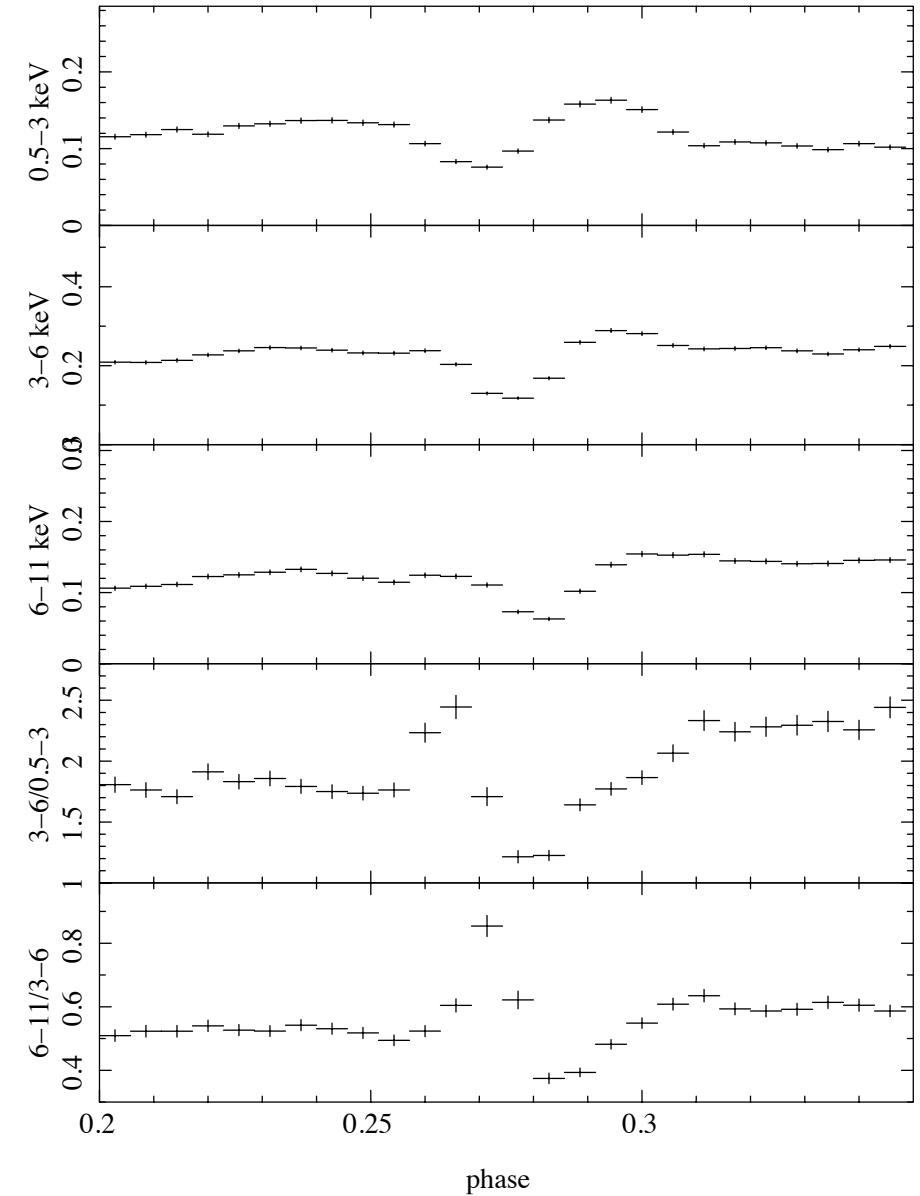

Fig. 2. Same as Fig. 1, but zoomed around the pronounced narrow feature at phase $\sim 0.27$.

at $90 \%$ c.l, which means that no significant ionization could be measured. All absorption components used the element abundances reported by Wilms et al. (2000) and cross sections taken from Verner et al. (1996).

We completed the spectral model with two Gaussian emission lines to take into account the neutral $\mathrm{K} \alpha$ iron line at $6.4 \mathrm{keV}$ and the $\mathrm{K} \beta$ line at $7.05 \mathrm{keV}$. Additional lines corresponding to higher ionization stages (Fe XVI) of the iron ions were detected only in the 2007 Suzaku data, when the source was significantly brighter. In this observation, we also detected the Si XIV at $2.5 \mathrm{keV}$ and the S XV and $3.2 \mathrm{keV}$, similarly to Naik et al. (2013), while the S XIII line at $2 \mathrm{keV}$ lies in the boundary of our energy selection windows and cannot be effectively constrained. The width of all Gaussian lines was fixed to zero in those fits where no significant broadening could be measured and was left free to very in all other cases (see Table 2). To account for calibration uncertainties, we added a $1 \%$ systematics during the fits. Owing to known Suzaku calibration problems, we left the power-law photon index in the spectrum extracted from the back-illuminated CCD (XIS1) free to vary with respect to those measured from the front-illuminated chips and from the PIN instrument. A minor difference was obtained ( $\sim 0.05)$, but this was enough to cause a significant improvement in the resulting $\chi^{2}$. In the 2007 observation, we obtained $\Delta \chi^{2}=97$ (from 462 to 461 d.o.f.). when we allowed different power-law slopes. For the 2012 data, the improvement was $\Delta \chi^{2}=109$ passing from 383 to 382 d.o.f. 


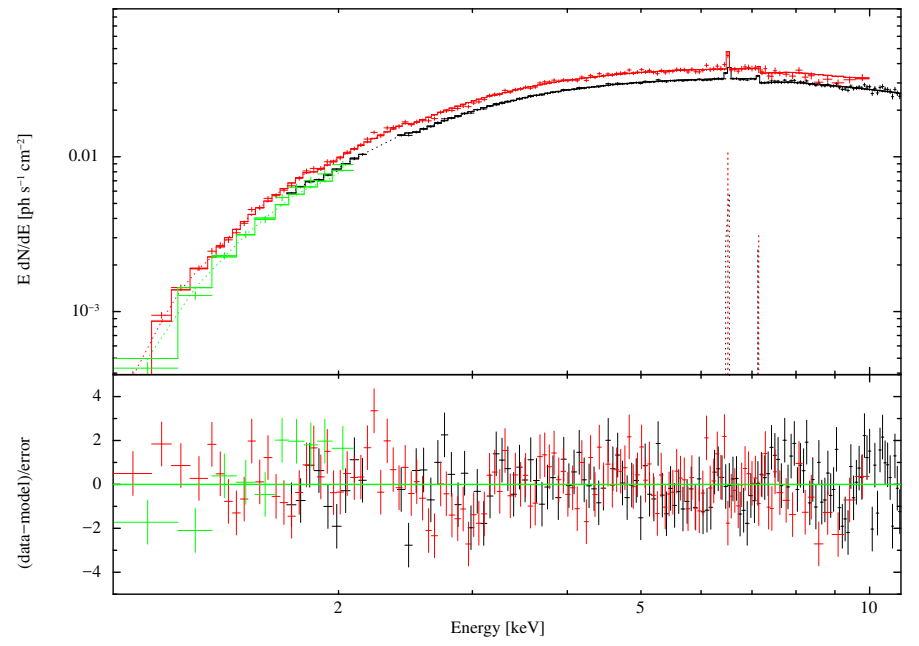

Fig. 3. Phase-averaged unfolded energy spectrum of the XMM-Newton observation carried out in 2014. All best-fit parameters are reported in Table 2. EPIC-pn (black), EPIC-MOS (red), and RGS (green) data have been rebinned for plotting purposes.

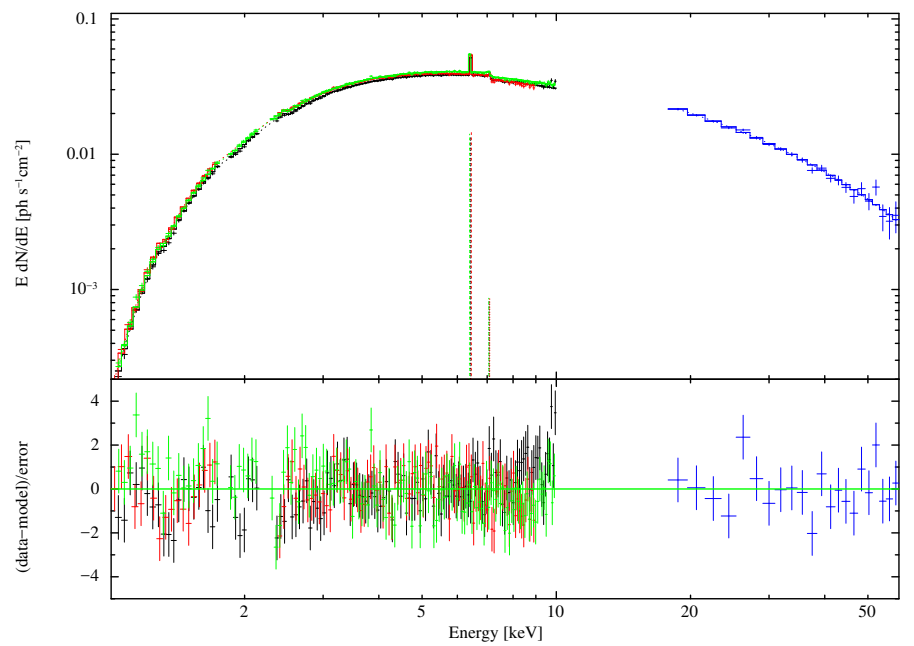

Fig. 4. Same as Fig. 3, but for the Suzaku data collected in 2012. XIS0 (black), XIS1 (red), XIS3 (green), and HXD-PIN data (blue) have been rebinned for plotting purposes.

A comparison between the results obtained with the above fits to the XMM-Newton and Suzaku data collected in 2012 reveals that there is a good agreement with the properties of the partial absorber, while the continuum parameters show some significant deviations. We are currently unable to determine whether the properties of the source are intrinsically different or if this can be due to inter-calibration issues of the instruments. We verified that the different energy ranges covered by the two facilities do not affect this outcome, as neglecting the PIN data in Suzaku only resulted in a slightly more poorly constrained cutoff and folding energies. We comment on this point in greater detail in Sect. 6.

All best-fit parameters are reported in Table 2. Crosscalibration constants were introduced in all fits and fixed to unity for the EPIC-pn and the XIS0 because we used these instruments as references for the XMM-Newton and Suzaku observations, respectively. All spectral fits were performed by using XSPEC v12.8.1g (Arnaud 1996).

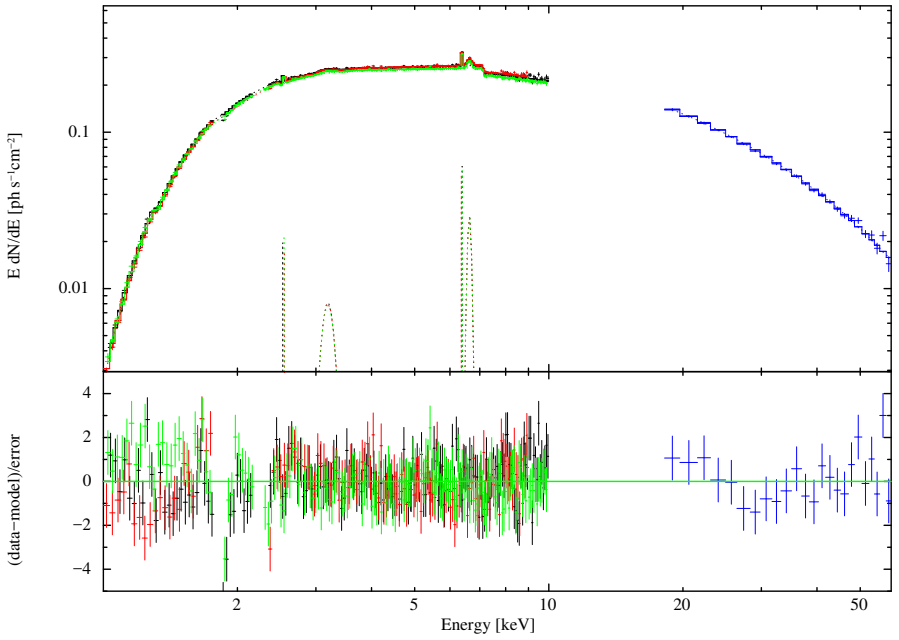

Fig. 5. Same as Fig. 3, but for the Suzaku data collected in 2007. XIS0 (black), XIS1 (red), XIS3 (green), and HXD-PIN (blue) data have been rebinned for plotting purposes.

\section{Phase-resolved spectroscopy}

To perform the phase-resolved spectral analysis of the $X M M-N e w t o n$ observation, we divided the source spin period into 128 phase bins and extracted the corresponding EPIC-pn and MOS1 spectra. We restricted the analysis of the 2012 and 2007 Suzaku observations to 20 phase bins because of the limited timing resolution of these data. The corresponding XIS, PIN, and GSO spectra were then extracted. All spectra were grouped with at least 20 photons per energy bin in addition to the optimal oversampling of the energy response to guarantee the usability of the $\chi^{2}$ statistical test. In all cases, a randomization of the arrival time of each photon within the instrumental time bin was applied to avoid any discretization problems. We fit all spectra with the same model as employed in Sect. 4, but freezing a few parameters that could not be constrained in these lower statistic spectra to the values measured from the phase-averaged analysis. In particular, we fixed the Galactic column density and the energy and widths of all iron lines (these are not expected to change as a function of the NS spin period). We also removed the iron $\mathrm{K} \beta$ line from the fit because this feature could not be detected in the phase-resolved spectra.

Performing fits to all data with the above assumptions still resulted in a number of poorly constrained spectral parameters. In all phase-resolved XMM-Newton spectra, we fixed the cutoff and folding energies to the average values. Only in spectra for which the source flux was low did the column density of the partially covering component turn out to be virtually unconstrained and was therefore fixed to its average value.

All results of the phase-resolved spectral analysis are summarized in Figs. 6-8. The sharp feature described in Sect. 4 cannot be detected in the Suzaku pulse profiles because of the lower timing resolution. The analysis of the XMM-Newton data revealed significant spectral changes corresponding to this feature. Our analysis suggests that there is a remarkable increase in the absorption column density during the ingress into the feature, combined with a strong hardening of the power-law slope. After the ingress, the absorption column density decreases and the spectra soften. The partial covering parameters corresponding to the out-of-feature values are recovered shortly before egress from the feature. The other spectral variations observed along the pulse profile of the source agree reasonably well with those measured from the 2012 Suzaku data. The difference in the spectral 
Table 2. Spectral parameters obtained from the best fits to the XMM-Newton and Suzaku phase-averaged data.

\begin{tabular}{|c|c|c|c|}
\hline & XMM-Newton & Suzaku 2012 & Suzaku 2007 \\
\hline $\begin{array}{l}E_{\mathrm{FeK} \alpha}[\mathrm{keV}] \\
N_{\mathrm{FeK} \alpha}\left[\mathrm{ph} \mathrm{s}^{-1} \mathrm{~cm}^{-2}\right]\end{array}$ & $\begin{array}{c}6.50 \pm 0.01 \\
(1.0 \pm 0.1) \times 10^{-4}\end{array}$ & $\begin{array}{c}6.41 \pm 0.01 \\
(1.8 \pm 0.2) \times 10^{-4}\end{array}$ & $\begin{array}{c}6.38 \pm 0.02 \\
(6 \pm 1) \times 10^{-4}\end{array}$ \\
\hline$N_{\mathrm{FeK} \beta}{ }^{a}\left[\mathrm{ph} \mathrm{s}^{-1} \mathrm{~cm}^{-2}\right]$ & $<2 \times 10^{-5}$ & $(4 \pm 1) \times 10^{-5}$ & - \\
\hline$E_{\mathrm{FexxvI}}[\mathrm{keV}]$ & - & - & $6.63 \pm 0.02$ \\
\hline$\sigma_{\mathrm{FexxvI}}[\mathrm{keV}]$ & - & - & $0.07 \pm 0.03$ \\
\hline$N_{\mathrm{FexxvI}}\left[\mathrm{ph} \mathrm{s}^{-1} \mathrm{~cm}^{-2}\right]$ & - & & $(8 \pm 2) \times 10^{-4}$ \\
\hline$E_{\mathrm{Si} \mathrm{XIV}}[\mathrm{keV}]$ & - & - & $2.53_{-0.04}^{+0.10}$ \\
\hline$N_{\text {Si XIV }}\left[\mathrm{ph} \mathrm{s}^{-1} \mathrm{~cm}^{-2}\right]$ & - & & $(5 \pm 2) \times 10^{-4}$ \\
\hline$E_{\mathrm{Sxv}}[\mathrm{keV}]$ & - & - & $3.19 \pm 0.04$ \\
\hline$\sigma_{\mathrm{S} \mathrm{xv}}[\mathrm{keV}]$ & - & - & $0.09_{-0.03}^{+0.04}$ \\
\hline$N_{\mathrm{S} \mathrm{Xv}}\left[\mathrm{ph} \mathrm{s}^{-1} \mathrm{~cm}^{-2}\right]$ & - & - & $(8 \pm 3) \times 10^{-4}$ \\
\hline$N_{\mathrm{H}}\left[10^{22} \mathrm{~cm}^{-2}\right]$ & $2.84 \pm 0.07$ & $3.13 \pm 0.05$ & $2.97 \pm 0.02$ \\
\hline$N_{\mathrm{H}, \mathrm{pc}}\left[10^{22} \mathrm{~cm}^{-2}\right]$ & $8.6 \pm 0.4$ & $8.1 \pm 0.4$ & $124 \pm 18$ \\
\hline$f$ & $0.59 \pm 0.01$ & $0.58 \pm 0.01$ & $0.24 \pm 0.03$ \\
\hline$\Gamma$ & $1.17 \pm 0.02$ & $1.33 \pm 0.03$ & $1.31 \pm 0.01$ \\
\hline$\Gamma_{\mathrm{XIS1} 1}$ & - & $1.38 \pm 0.03$ & $1.27 \pm 0.01$ \\
\hline$E_{\mathrm{C}}[\mathrm{keV}]$ & $7.9 \pm 0.2$ & $7.0 \pm 0.3$ & $6.8_{-0.1}^{+0.2}$ \\
\hline$E_{\mathrm{F}}[\mathrm{keV}]$ & $18 \pm 2$ & $26 \pm 1$ & $20.9 \pm 0.4$ \\
\hline $\operatorname{Flux}_{\mathrm{PL},(0.5-10 \mathrm{keV})^{b}}$ & $5.72 \pm 0.07$ & $7.53 \pm 0.13$ & $59_{-2}^{+3}$ \\
\hline$\chi_{\text {red }}^{2}$ (d.o.f.) & $1.158^{c}(456)$ & $1.091^{c}(382)$ & $1.106^{c}(461)$ \\
\hline$C_{\mathrm{MOS} 1}$ & $1.157 \pm 0.005$ & - & - \\
\hline$C_{\mathrm{RGS}}$ & $0.27^{d} \pm 0.01$ & - & - \\
\hline$C_{\mathrm{XIS} 1}$ & - & $1.045 \pm 0.005$ & $0.995 \pm 0.003$ \\
\hline$C_{\mathrm{XIS} 3}$ & - & $1.054 \pm 0.004$ & $0.968 \pm 0.002$ \\
\hline$C_{\mathrm{PIN} / \mathrm{GSO}}$ & - & $1.18 \pm 0.03$ & $1.08 \pm 0.04$ \\
\hline
\end{tabular}

Notes. ${ }^{(a)}$ The centroid energy is fixed to be $0.65 \mathrm{keV}$ higher than that of the $\mathrm{K} \alpha$ line. The widths of the Gaussian lines are fixed to zero when not explicitly reported. ${ }^{(b)}$ The flux of the power-law component without exponential cutoff and absorption is a fit parameter expressed in units of $10^{-10} \mathrm{erg} \mathrm{s}^{-1} \mathrm{~cm}^{-2}$. (c) A $0.5 \%$ (1\%) systematic error is added in quadrature for XMM-Newton (Suzaku) data. ${ }^{(d)}$ This small («1) inter-calibration constant is due to a technical feature of XSPEC and reflects the smaller energy range of the RGS response, as compared to the EPIC cameras.

parameters measured between the 2007 and 2012 Suzaku data has been discussed by Naik \& Jaisawal (2015). We briefly comment on this point in Sect. 6.

\section{Discussion}

We reported on the first XMM-Newton observation of EXO 2030+375 performed in 2014 during the rise to one of the source type I X-ray outbursts. The large collecting areas and good timing resolution of the EPIC cameras onboard $X M M$-Newton allowed us to study the source pulse profile with high accuracy and measure spectral energy variations as a function of the source spin phase.

The spectral energy distribution of EXO $2030+375$ in the XMM-Newton energy band could be well described by using a phenomenological model comprising a power law with an exponential cutoff at high energies and two absorption components. One of these takes into account the Galactic and circum-binary obscuring material in the direction of the X-ray source (the expected Galactic column density in the direction of EXO $2030+375$ is $\sim 0.9 \times 10^{22} \mathrm{~cm}^{-2}$, Kalberla et al. 2005); while the second is introduced to describe the effect of a spindependent partially covering medium in the closest proximities of the NS. Even though the latter is expected to be ionized as a result of the relatively high impinging X-ray flux from the compact object, we were unable to reveal any spectral signature in the XMM-Newton spectra that could be related to the ionization, as revealed by the tight upper limit on the ionizations parameter, obtained using the warmabs model. This is somewhat unexpected based on simple calculations. When we consider the source intrinsic X-ray luminosity measured by XMM-Newton, $L_{\mathrm{X}} \sim 1.1 \times 10^{37} \mathrm{erg} / \mathrm{s}$ (derived from the unabsorbed $0.1-100 \mathrm{keV}$ flux $\sim 1.8 \times 10^{-9} \mathrm{erg} / \mathrm{s} / \mathrm{cm}^{2}$ at $7.1 \mathrm{kpc}$ ), we can estimate the average mass-accretion rate as $\dot{m}=5.8 \times 10^{16} \mathrm{~g} / \mathrm{s}$ (assuming that all the potential energy of the accreting matter is converted into $\mathrm{X}$-ray radiation). For an NS with a mass (radius) of $1.4 M_{\odot}$ $(10 \mathrm{~km})$ and a dipole magnetic field of $5.4 \times 10^{12} \mathrm{G}$ (as in EXO 2030+375; see, e.g., Klochkov et al. 2007), the accretion column is expected to have a radius of $\sim 300 \mathrm{~m}$ on the stellar surface (we considered that the accretion flow is uniform within the accretion column and that the latter intercepts the inner boundary of the accretion disk at the Alfvén radius given by Eq. (6.19) of Frank et al. 2002). In the free-fall approximation, the particle density is thus expected to be of about $10^{20} \mathrm{~cm}^{-3}$ close to the NS surface and to decrease by a factor of two $3 \mathrm{~km}$ above (reaching a minimum of $\sim 10^{12} \mathrm{~cm}^{-3}$ at the Alfvén radius). As the ionization 


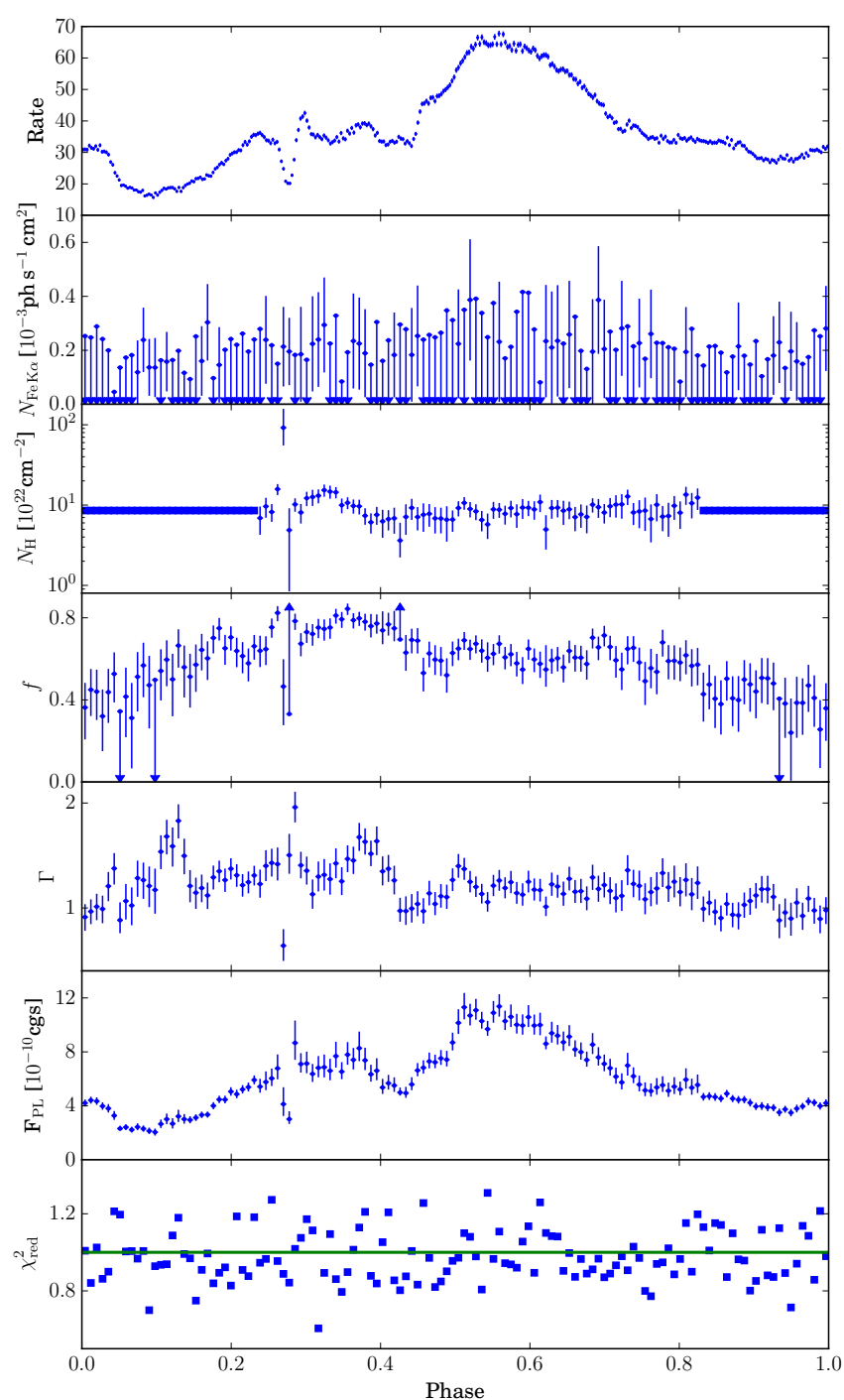

Fig. 6. Phase-resolved spectral parameters obtained from the fits to the XMM-Newton data. The pulse profile is extracted in 350 bins, while the spectra are extracted in 128 bins $(0.5-10 \mathrm{keV})$. Filled squares in the parameter panels indicate a frozen parameter value. The reduced $\chi^{2}$ is computed with a number of degrees of freedom of between 99 and 199 , depending on the statistics of the different spectra. Uncertainties are reported at $90 \%$ c.l. for all parameters.

parameter $\xi=\frac{L_{\mathrm{X}}}{n d^{2}}$ (where $d$ is the distance of the medium from the illuminating source, Kallman et al. 2004) is larger than $10^{5}$ up to the Alfvén radius, the bulk of the accreting material around the NS magnetic field lines is expected to be highly ionized. We note that this issue is also observed in the similar system KS 1947+300 (Ballhausen et al. 2016), for example.

A way to solve this apparent problem is to assume that either the bulk of the X-ray radiation from the NS is highly beamed and does not (always) sufficiently illuminate the accretion stream, or the material in the accretion stream is inhomogeneous and overdense compared to our previous estimate. The assumption of an inhomogeneous and overdense accretion flow seems to agree with the low covering fraction measured by our spectral analysis (see Table 2 and Figs. 7 and 8) and it is known to be more likely to occur in presence of complex accretion geometries, owing to the tilt between the magnetic and rotational axes and the coupling between the NS magnetosphere with the

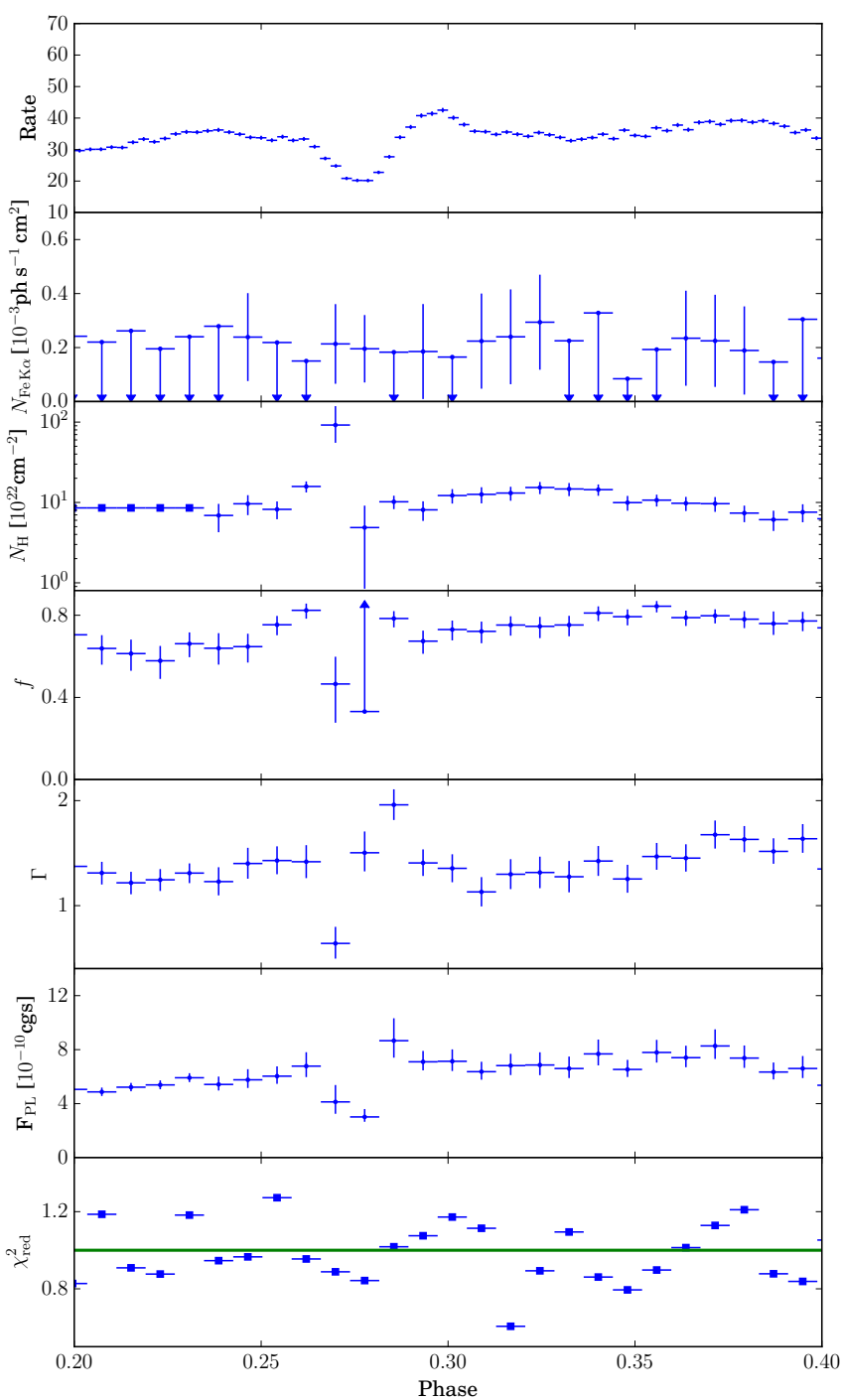

Fig. 7. Zoom into the phase-resolved spectral analysis of Fig. 6 around the sharp V-shaped feature described in Sect. 4.

accretion disk (see, e.g., Meszaros 1984). As an example, hollow accretion columns would naturally lead to higher densities. It has been also suggested in the literature that the typical variability of EXO 2030+375 and other high-mass X-ray binaries could be related to a clumpy (and thus strongly inhomogeneous) stream being accreted onto the NS (Klochkov et al. 2011). A beamed radiation would certainly not be surprising in a young X-ray pulsar because of the well-known angular dependence of the scattering cross-section in presence of a strong magnetic field. The latter can lead to the X-ray radiation being preferentially emitted along the direction of the stream at low luminosities (pencil beam) or perpendicular with respect to the stream (fan beam) at higher luminosities. The second possibility would be favorable to reduce the ionization state of the accreting material, but we note that a combination of simultaneous fan and pencil beams might also be a possibility (see, e.g., Leahy 2004).

By analyzing two Suzaku observations of EXO 2030+375 during two type I outbursts that occurred in 2007 and 2012, we showed that the spectral model used to fit the phaseaveraged XMM-Newton data is also suitable to describe the X-ray emission over a broader energy range (1-100 keV). Although the 2012 Suzaku observation caught the source at a 


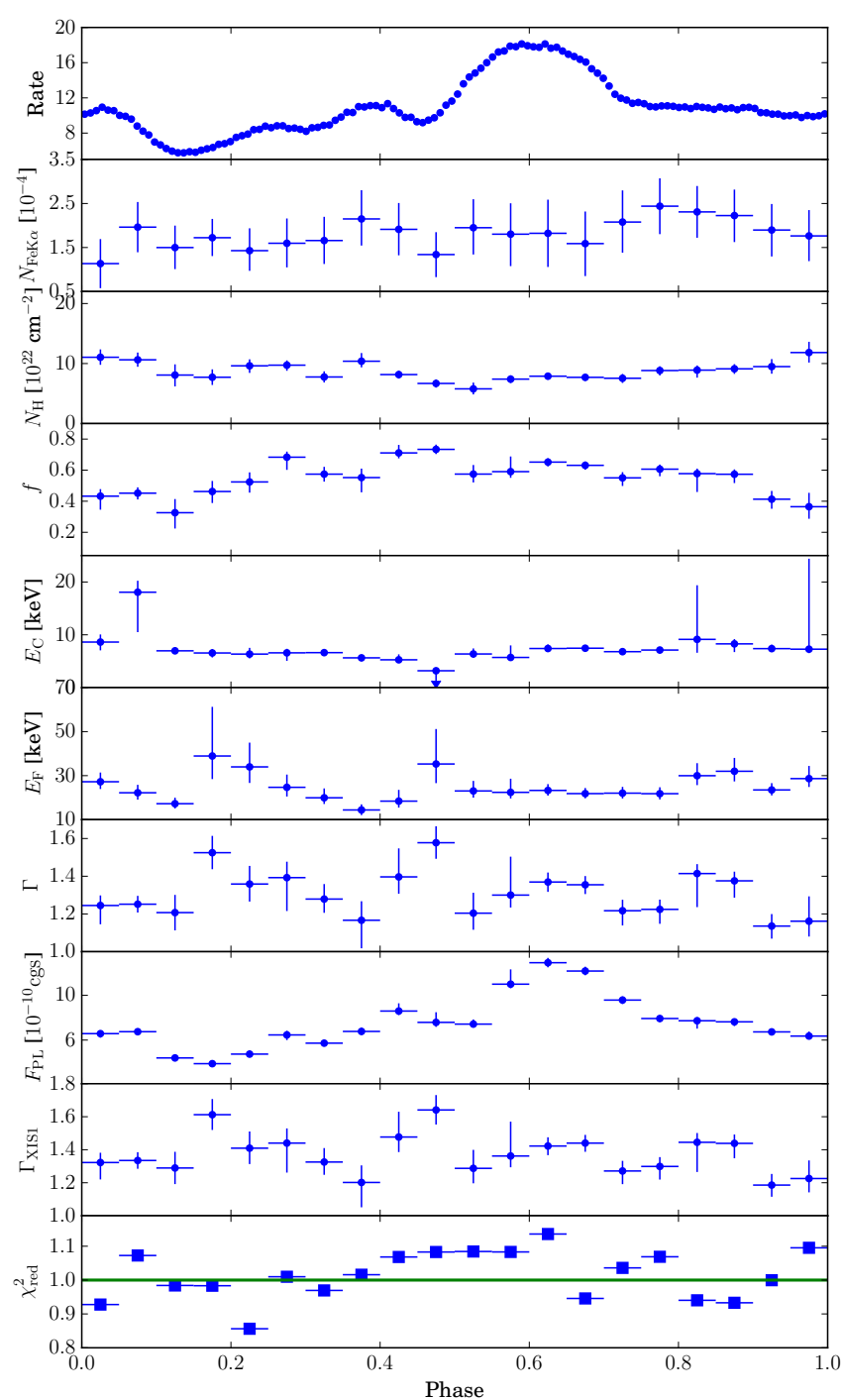

Fig. 8. Phase-resolved spectral parameters obtained from the Suzaku observation carried out in 2012. The pulse profile is extracted in the $0.5-10 \mathrm{keV}$ energy range using 128 phase bins (a randomization of the photon arrival times within the time-resolution unit has been applied in all cases). Twenty phase-resolved spectra have been extracted because the timing resolution of the Suzaku data is lower than those of the $X M M$-Newton data. The normalizations of the iron line are expressed in units of $10^{-4} \mathrm{ph} \mathrm{s}^{-1} \mathrm{~cm}^{-2}$. The reduced $\chi^{2}$ is computed with a number of degrees of freedom of between 323 and 378, depending on the statistics of the different spectra.

similar luminosity as the one recorded from the XMM-Newton data, a number of differences in the spectral parameters were measured in the analysis carried in Sects. 4 and 5. Owing to intercalibration uncertainties between the XMM-Newton and Suzaku instrument, we cannot rule out that some of these discrepancies were instrumental, but it is also possible that some of the differences in spectral parameters are the result of different physical conditions in the accretion columns, since the two outbursts are separated by many orbital revolutions. A more reliable comparison of the source high-energy spectral distribution in different outbursts can be carried out between the results obtained from the fits performed on the Suzaku data collected in 2012 and 2007. Table 2 shows a dramatic increase in the column density of the partial covering component with luminosity and an

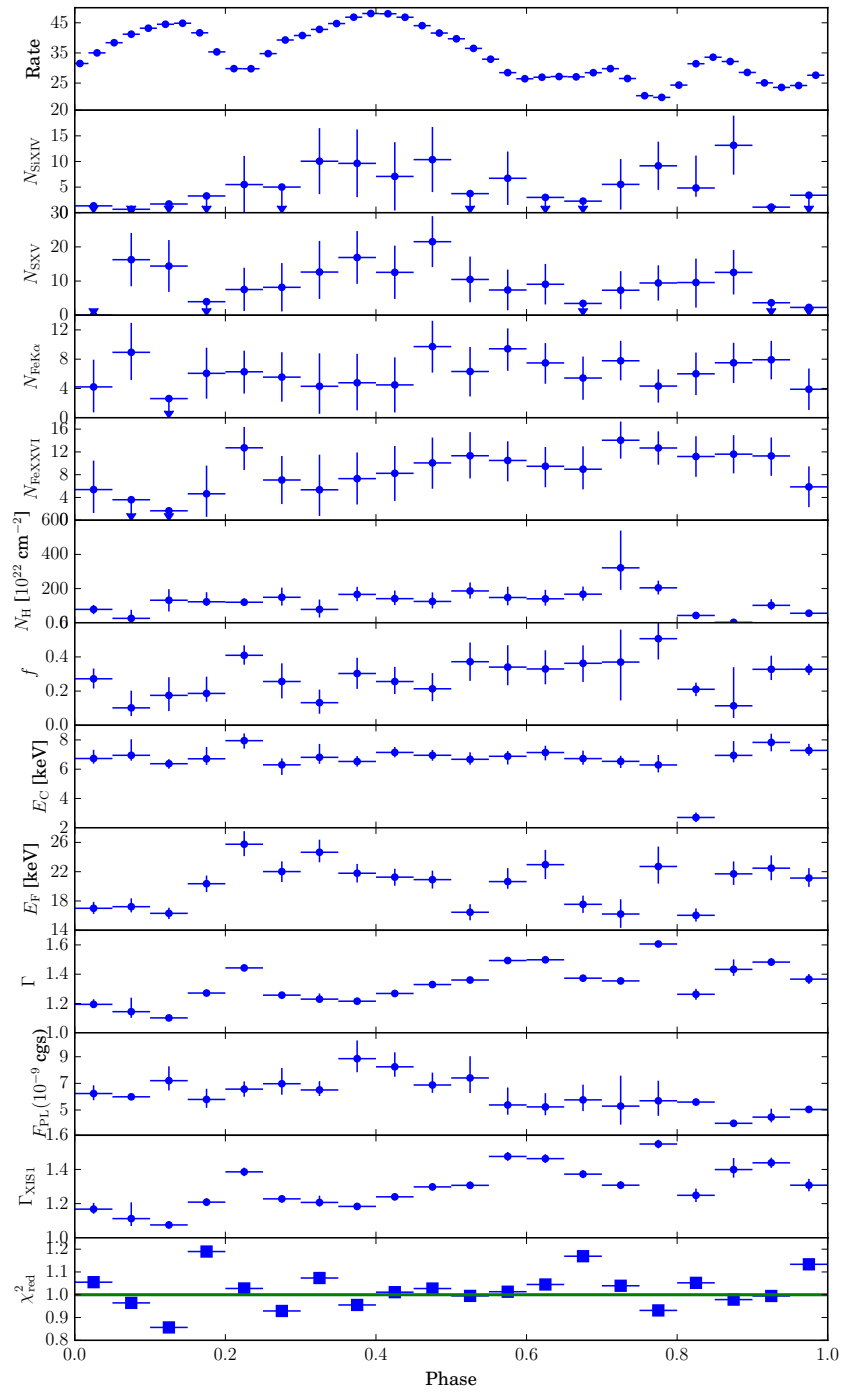

Fig. 9. Same as Fig. 8, but for the Suzaku observation carried out in 2007. The reduced $\chi^{2}$ is computed with a number of degrees of freedom of between 402 and 435, depending on the statistics of the different spectra.

opposite behavior for the covering fraction (as also noted by Naik et al. 2013; Naik \& Jaisawal 2015). The fluorescence lines corresponding to higher ionization states of the iron ions and by sulfur and silicon become detectable in the higher luminosity observation as a result of the increased ionizing X-ray flux. Figure 9 shows that the neutral iron emission line intensity is roughly constant along the pulse phase for all datasets, while the lines of the higher ionization stages show significant changes, which are suggestive of variable illuminations effects. This would be consistent with the idea of an inhomogeneous medium and an asymmetric radiation beam.

From the phase-resolved spectral analysis of the $X M M$-Newton observation, we studied with unprecedented detail spectral changes of the source emission in the soft $\mathrm{X}$-ray energy band as a function of the spin phase. The overall behavior of the main parameters is relatively similar in the XMM-Newton and the Suzaku data collected at comparable luminosities. In the EPIC-pn data, we revealed a remarkable spectral variation during the ingress and egress of a sharp 


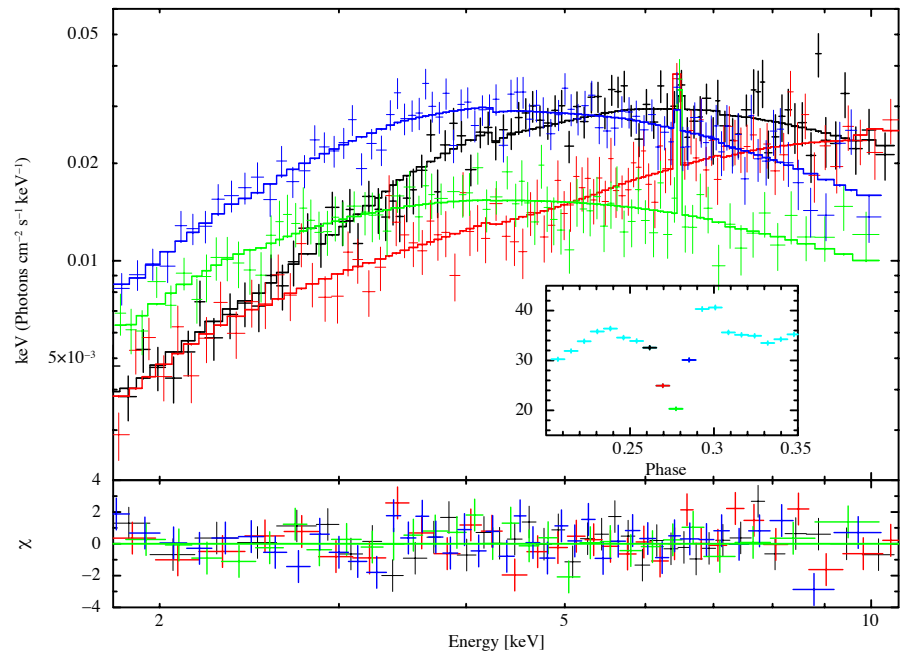

Fig. 10. Upper panel: phase-resolved deconvolved energy spectra extracted from the XMM-Newton observation during the V-shaped feature analyzed in Sect. 5. The best-fit model for all spectra is the one described in Sect. 4. The different selected phases for which the spectra are extracted are represented with the same color in the zoomed pulse profile in the inset (cyan points represent the pulse profile data for which no spectrum is displayed). Only EPIC-pn data are shown for clarity. The lower panel shows the residuals from the fit.

V-shaped feature profile, which was never detected before (see also Fig. 10). We note that a different dip-like structure in the pulse profile of EXO $2030+375$ was also reported by Klochkov et al. (2008) using INTEGRAL data collected around the maximum of the source giant outburst that occurred in 2006. This feature appeared only above $10 \mathrm{keV}$ and vanished above $70 \mathrm{keV}$. Therefore, it could have a completely different origin compared to that detected by XMM-Newton in the soft $\mathrm{X}$-rays. According to the fits, this spectral variability can be ascribed mostly to the strong increase in the column density of the ionized absorber during the ingress into the feature and to the hardening of the spectrum (changes in the power-law photon index $\Gamma$ from $\sim 0.7$ to 2 are observed across the $\mathrm{V}$-shaped feature). The source seems then to recover the usual spectral parameters after the egress from the feature. As the density of the accretion stream near the NS is $\sim 10^{20} \mathrm{~cm}^{-3}$, variations of about $10^{22} \mathrm{~cm}^{-2}$ in the column density can be expected if the line of sight to the observer in the direction of the X-ray source is obscured by just a few meters of stratified accreting material. This part of the accretion column can also become a source of reprocessed radiation, whose intensity and hardness can change depending on the viewing angle in different the phase-resolved spectra. We therefore interpret the $\mathrm{V}$-shaped feature as being due to the obscuration of the compact emitting region on the NS surface by the accretion column that passes in front of the observer line of sight and produces an enhanced scattered radiation at the egress. As the obscuration occurs on a timescale of $\sim 1 / 128 P_{\text {spin }} \sim 0.3 \mathrm{~s}$, which corresponds to a linear scale of $\sim 500 \mathrm{~m}$ onto the NS surface, the XMM-Newton observation also provides constraints on the lateral extension of the accretion column in EXO 2030+375. According to this interpretation, this would mean that we directly glance through the NS accretion column during the short time interval corresponding to the $\mathrm{V}$-shaped feature in the source pulsed profile.

This V-shaped feature could not be verified in the 2012 Suzaku data because of their limited timing resolution. At odds with Naik \& Jaisawal (2015), our phase-resolved spectral analysis did not reveal any significant increase in the absorption column density at phase $0.7-0.9$, corresponding to the second peak in the hard X-ray pulse profile. We also inspected the 2007 Suzaku data for the presence of absorption-driven structures in the pulse profile. In Fig. 9, we noted a relatively broad dip structure at phase 0.75 , which could be the high-luminosity counterpart of the dip observed in the XMM-Newton data set. In this case, we should also assume that the peak at phase 0.15 in the 2007 Suzaku data corresponds to the main peak of the source pulse profile observed by XMM-Newton at phase 0.6. The dip structure in the 2007 Suzaku data spans $~ 1 / 20$ of the spin phase, translating into a projected scale on the NS surface of about $3 \mathrm{~km}$. Based on a simple scaling relation of the NS hot-spot angular size on the NS surface $\theta$ as function of the X-ray luminosity $\left(\theta \propto L_{\mathrm{X}}^{1 / 7}\right.$, Lamb et al. 1973), we would expect an increase of only about $40 \%$ in the lateral size of the NS accretion column between the XMM-Newton and the 2007 Suzaku observations. A more likely possibility is that the dip structure in the 2007 Suzaku data is due to the obscuration effect of a larger section of the accretion stream farther above the NS surface. Assuming a dipolar field, an increase in the angular size of the absorber by a factor of 6 would imply that the obscuring region is located at about 40 stellar radii from the NS surface (without accounting for any relativistic effect). The results displayed in Fig. 9 show a marginal indication of an increase in the local absorption column density and covering fraction at the phase corresponding to the dip, further supporting this interpretation.

\section{Conclusion}

The discovery of a sharp and narrow dip-like feature in the soft X-ray pulse profile of EXO $2030+375$ during a typical type I X-ray outburst allowed us to gain a novel insight into the physical properties of the accretion flow in this object. The presence of such feature is so far unique in all known high-mass X-ray binaries hosting strongly magnetized stars (to the best of our knowledge). Additional investigations on other similar systems with $\mathrm{X}$-ray instruments endowed with a good timing resolution and large effective areas at $\sim 10 \mathrm{keV}$ could complement the existing legacy data, typically available only at higher luminosities and harder X-rays, and allow us to probe the accretion geometry and magnetic field topology of these systems in greater detail.

A model comprising a phenomenological Comptonization continuum and a combination of homogeneous and inhomogeneous absorbers was shown to provide a reasonably good fit to the XMM-Newton data of EXO 2030+375 and to the spectra of the source with broader energy coverage provided by the Suzaku observations. Exploiting the suitability of such a model to fit the X-ray spectra of other BeXRBs might permit us to achieve a more homogeneous description of the high-energy emission from these sources.

Acknowledgements. This work is based on observations obtained with XMM-Newton (OBSID 74524), an ESA science mission with instruments and contributions directly funded by ESA Member States and the USA (NASA) and of data obtained from the Suzaku satellite (OBSIDs 402068010 and 407089010), a collaborative mission between the space agencies of Japan (JAXA) and the USA (NASA). P.P. and A.A.Z. have been supported in part by the Polish National Science Centre grants 2012/04/M/ST9/00780, 2013/10/M/ST9/00729, and 2015/18/A/ST/00746. L.D. is supported by the Bundesministerium für Wirtschaft und Technologie through the Deutsches Zentrum für Luft- und Raumfahrt (grant FKZ 50 OG 1602). 


\section{References}

Arnaud, K. A. 1996, Astronomical Data Analysis Software and Systems V, 101, 17

Ballhausen, R., Kühnel, M., Pottschmidt, K., et al. 2016, A\&A, 591, A65

Becker, P. A., \& Wolff, M. T. 2007, ApJ, 654

Bildsten, L., Chakrabarty, D., Chiu, J., et al. 1997, ApJS, 113, 367

Coe, M. J., Payne, B. J., Longmore, A., \& Hanson, C. G. 1988, MNRAS, 232, 865

Cutri, R. M., Skrutskie, M. F., van Dyk, S., et al. 2003, VizieR Online Data Catalog: II $/ 246$

Farinelli, R., Ceccobello, C., Romano, P., \& Titarchuk, L. 2012, A\&A, 538, A67

Farinelli, R., Ferrigno, C., Bozzo, E., \& Becker, P. A. 2016, A\&A, 591, A29

Ferrigno, C., Becker, P. A., Segreto, A., Mineo, T., \& Santangelo, A. 2009, A\&A, 498,825

Ferrigno, C., Bozzo, E., Papitto, A., et al. 2014, A\&A, 567, A77

Frank, J., King, A., \& Raine, D. J. 2002, Accretion Power in Astrophysics: Third Edition (Cambridge University Press)

Kaastra, J. S., \& Bleeker, J. A. M. 2016, A\&A, 587, A151

Kalberla, P. M. W., Burton, W. B., Hartmann, D., et al. 2005, A\&A, 440, 775

Kallman, T. R., Palmeri, P., Bautista, M. A., Mendoza, C., \& Krolik, J. H. 2004, ApJS, 155, 675

Klochkov, D., Horns, D., Santangelo, A., et al. 2007, A\&A, 464, L45

Klochkov, D., Santangelo, A., Staubert, R., \& Ferrigno, C. 2008, A\&A, 491, 833

Klochkov, D., Staubert, R., Santangelo, A., Rothschild, R. E., \& Ferrigno, C. 2011, A\&A, 532, A126
Kühnel, M., Müller, S., Kreykenbohm, I., et al. 2013, A\&A, 555, A95

Lamb, F. K., Pethick, C. J., \& Pines, D. 1973, ApJ, 184, 271

Leahy, D. A. 2004, MNRAS, 348, 932

Meszaros, P. 1984, Space Sci. Rev., 38, 325

Meszaros, P., \& Nagel, W. 1985, ApJ, 298, 147

Meszaros, P., \& Riffert, H. 1988, ApJ, 327, 712

Naik, S., \& Jaisawal, G. K. 2015, RA\&A, 15, 537

Naik, S., Paul, B., \& Ali, Z. 2011, ApJ, 737, 79

Naik, S., Maitra, C., Jaisawal, G. K., \& Paul, B. 2013, ApJ, 764, 158

Nowak, M. A., Hanke, M., Trowbridge, S. N., et al. 2011, ApJ, 728, 13

Parmar, A. N., White, N. E., \& Stella, L. 1989, ApJ, 338, 373

Reig, P., \& Coe, M. J. 1999, MNRAS, 302, 700

Sasaki, M., Klochkov, D., Kraus, U., Caballero, I., \& Santangelo, A. 2010, A\&A, 517, A8

Stella, L., White, N. E., \& Rosner, R. 1986, ApJ, 308, 669

Suchy, S., Pottschmidt, K., Wilms, J., et al. 2008, ApJ, 675, 1487

Verner, D. A., Ferland, G. J., Korista, K. T., \& Yakovlev, D. G. 1996, ApJ, 465, 487

Walter, R., Lutovinov, A. A., Bozzo, E., \& Tsygankov, S. S. 2015, A\&ARv, 23, 2

Wilms, J., Allen, A., \& McCray, R. 2000, ApJ, 542, 914

Wilson, C. A., Finger, M. H., Coe, M. J., Laycock, S., \& Fabregat, J. 2002, ApJ, 570,287

Wilson, C. A., Fabregat, J., \& Coburn, W. 2005, ApJ, 620, L99

Wilson, C. A., Finger, M. H., \& Camero-Arranz, A. 2008, ApJ, 678, 1263 